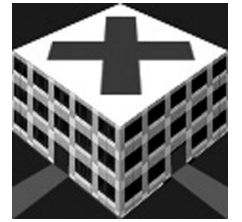

\title{
MMMII Use of Interpreters by Physicians for Hospitalized Limited English Proficient Patients and Its Impact on Patient Outcomes
}

\author{
Lenny López, MD, MPH ${ }^{1,2}$, Fátima Rodriguez, MD, MPH², Diego Huerta, $B A^{3}$, Jane Soukup, $\mathrm{MSC}^{2}$, and \\ Leroi Hicks, MD, MPH
}

'Department of General Medicine, Mongan Institute for Health Policy, and Disparities Solutions Center, Massachusetts General Hospital, Boston, MA, USA; ' 2 Department of Medicine, Brigham and Women's Hospital, Boston, MA, USA; ${ }^{3}$ Harvard College, Harvard University, Cambridge, MA, USA; ${ }^{4}$ Department of Medicine, Christiana Care Health System and Value Institute, Christiana Care Health System, Newark, DE, USA.

BACKGROUND: Few studies have examined the impact of inpatient interpreter use for limited English proficient (LEP) patients on length of stay (LOS), 30-day post discharge emergency department (ED) visits and 30-day hospital readmission rates for LEP patients.

METHODS: A retrospective cohort analysis was conducted of all hospitalized patients admitted to the general medicine service at a large academic center. For patients self-reported as LEP, use of interpreters during each episode of hospitalization was categorized as: 1) interpreter used by non-MD (i.e., nurse); 2) interpreter used by a nonHospitalist MD; 3) interpreter used by Hospitalist; and 4) no interpreter used during hospitalization. We examined the association of English proficiency and interpreter use on outcomes utilizing Poisson and logistic regression models.

RESULTS: Of 4,224 patients, 564 (13\%) were LEP. Of these LEP patients, $65.8 \%$ never had a documented interpreter visit, $16.8 \%$ utilized an interpreter with a non-MD, $12.6 \%$ utilized an interpreter with a nonHospitalist MD and $4.8 \%$ utilized an interpreter with a hospitalist present. In adjusted models, compared to English speakers, LEP patients with no interpreters had significantly shorter LOS. There were no differences in readmission rates and ED utilization between LEP and English-speaking patients. Compared to LEP patients with no interpreter use, those who had a physician use an interpreter had odds for a longer LOS, but there was no difference in odds of readmission or ED utilization.

CONCLUSION: Academic hospital clinician use of interpreters remains highly variable and physicians may selectively be using interpreters for the sickest patients.

KEY WORDS: limited English proficiency; interpreter use; length of stay; thirty-day readmissions.

$\mathrm{J}$ Gen Intern Med 30(6):783-9

DOI: $10.1007 / \mathrm{s} 11606-015-3213-\mathrm{x}$

(c) Society of General Internal Medicine 2015

Received July 22, 2014

Revised December 22, 2014

Accepted January 22, 2015

Published online February 10, 2015

\section{INTRODUCTION}

In 2011, over 25 million Americans self-rated themselves as speaking English less than "very well," and as a result, health care providers increasingly encounter patients with limited English proficiency (LEP). ${ }^{1,2}$ Effective communication between patient and medical staff is critical to diagnosis and treatment. Although the Joint Commission recommends the use of professional medical interpreters during medical encounters, many LEP patients do not have access to interpreters, and some clinicians continue to "get by" and do not use interpreters consistently. ${ }^{3,4}$ The use of professional medical interpreters is associated with increased patient satisfaction, quality of care, and improved disease-specific process measures and outcomes. ${ }^{5,6}$ The Institute of Medicine report Crossing the Quality Chasm states that the use of an interpreter is not only a quality, but also a patient safety imperative..$^{7-10}$

Most studies to date have focused on the impact of interpreters in the outpatient and emergency department (ED) settings. ${ }^{11,12}$ Few studies have explored the relationship between in-hospital physician use of interpreters and patient outcomes measured by length of stay (LOS) and readmission rates. Five prior studies have analyzed the outcomes of LEP hospitalized patients compared to English speakers, but only two of these studies examined the impact of interpreter use with conflicting results. ${ }^{12-16}$ The first study found no difference in LOS and 3-month ED utilization nor readmission rates with data from a large urban public hospital. ${ }^{14}$ The second study found that the use of professional interpretation at admission or both admission and discharge was associated with an increased LOS and decreased readmission rates compared to those LEP patients without professional interpretation. ${ }^{11}$ However, this study was limited by not having an English speaking comparison group.

Understanding the dynamic between LEP patients and their clinical providers is of utmost importance for understanding observed disparities in health care. ${ }^{17}$ To address the paucity of research on the impact of interpreters on inpatient outcomes, our paper examines two questions: 1) Are hospitalized LEP patients receiving interpreter services during hospital clinical encounters?; and 2) does the documented use of a professional medical interpreters by hospital physicians during the inpatient 
encounter impact LOS, 30-day emergency department (ED) visits after discharge and 30-day readmission rates for LEP patients compared to English speaking patients?

\section{METHODS}

We reviewed hospital administrative and interpreter services data for all hospitalized patients in 2009 admitted to the general medicine service at a large tertiary academic center $(n=4,224)$. We obtained electronic medical record data from the Research Patient Data Registry (RPDR), a research and administrative data source designed to identify patients who meet specified criteria through a query tool. ${ }^{18}$ Data elements obtained from the RPDR included patients' demographic characteristics (e.g., race/ethnicity, gender, age, primary language, and insurance status). Self-reported race and ethnicity from the RPDR was collected by registration staff using a standardized script at the time of hospital admission or at outpatient visits.

The study hospital provided language interpretation services in three modalities: in person, telephone, and video conferencing. There was $24 \mathrm{hrs} / 7$ days per week in person interpreter availability for the inpatient and Emergency Department for the top ten languages in Boston, with telephonic backup for less common languages. Self-reported preferred language was collected at registration by trained registrar personnel with standardized scripts. In addition, the nurse intake form on the hospital inpatient units collected and/or confirmed language preference at the time of admission. Internal hospital analyses have demonstrated high rates of language preference accuracy, primarily because the hospital registration data is part of a system wide registration database. At any point of patient contact, whether outpatient or inpatient, the collected language data was shared in one common database. In addition, the interpreter services department kept its own database of interpreter encounters, which was merged periodically with the registration system to assure accuracy. Finally, the City of Boston and Massachusetts state statistics reports state that the Boston limited English proficiency rate is on average $14.1 \%$, which is very similar to the rate of $13 \%$ presented in our paper. ${ }^{19,20}$ Thus, we have good internal and external validation of our cohort size, and the number of missed LEP cases is likely to be small.

For our analyses, self-reported LEP status was derived from the hospital interpreter service and the hospital admission registration databases. We used the hospital interpreter database and chart review for patients selfreported as LEP to ascertain who was present during the interpretation service. We categorized data regarding use of interpreters during each episode of hospitalization as: 1): interpreter used by non-physician (i.e., nurse); 2) interpreter used by a non-Hospitalist physician; 3 ) interpreter used by Hospitalist; and 4) no interpreter used during hospitalization.
We examined the association of English proficiency and interpreter use (English-speaking vs. each LEP category as listed above) on outcomes. Poisson models with log transformed LOS and logistic regression for the 30-day ED visits and 30-day readmission rates (both any and same diagnosis readmissions) were used with adjustment for patient clustering at the physician level using Proc Genmod in SAS version 9.3 (SAS Institute Inc, Cary, NC). Each model adjusted for patients' age, gender, race/ethnicity, insurance, principal discharge diagnosis, Charlson comorbidity score, type of admitting attending physician (Hospitalist vs. non-Hospitalist), and the patient's number of hospital admissions in the previous year as a baseline measure of healthcare utilization intensity. Similar stratified analyses were conducted among only LEP patients.

\section{RESULTS}

\section{Patient Characteristics}

Table 1 shows that of 4,224 patients, 564 (13\%) were LEP. Compared to English speakers, LEP patients were slightly older, more likely to be Hispanic and females, and a majority used either Medicare or Medicaid insurance. Even though LEP patients had a slightly higher Charlson Score, they had a shorter LOS, and a lower readmission rate. There were no differences between the two language categories in ED utilization after discharge or in the most frequent primary cardiac discharge diagnosis. Additionally, of the 564 LEP patients, most never had documented use of an interpreter. There were important differences in Charlson score by LEP interpreter use categories. Patients with an interpreter and a physician present $(n=71)$ had the highest Charlson score $(2.8 \pm 2.6)$, followed by those with no interpreter $(n=371$, Charlson score $(2.6 \pm 2.3))$, those with an interpreter and a Hospitalist present $(\mathrm{n}=27$, Charlson score $(2.3 \pm 1.8)$ ), and f,inally those with an interpreter and a nonphysician present $(\mathrm{n}=95$, Charlson score $(2.2 \pm 2.0)$ ).

\section{Hospital Outcomes by Patient Characteristics}

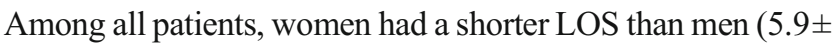
5.5 vs. $6.2 \pm 5.5)$. LOS by race was as follows: White $(6.4 \pm$ 5.8), Black (5.6 \pm 5.4$)$, Hispanic (4.9 \pm 3.7$)$, Asian (7.1 \pm 6.5$)$, and Other $(5.5 \pm 6.1)$. Both Hospitalist and non-Hospitalist services had a similar LOS (6.0 \pm 5.5 vs. $6.1 \pm 5.8)$. LEP patients who had an interpreter and a physician present had the longest LOS $(7.3 \pm 7.5)$, followed by those who had a hospitalist present $(6.5 \pm 4.6)$. Those who had an interpreter present with a non-physician present had the shortest LOS $(4.7 \pm 2.6)$, similar to those with no interpreter use recorded $(4.9 \pm 3.9)$. English speakers had a LOS of 6.2 \pm 5.7 .

Of 4,224 patients, there were only 765 readmissions for any diagnosis, of which 669 were English speakers (87.5\%) and 96 were LEP patients $(12.5 \%)(\mathrm{p}=0.47)$ (Table 1$)$. Of 765 readmissions, 260 readmissions were for the same diagnosis; 233 were English speakers (89.6 \%) and only 27 were LEP 
Table 1 Patient Characteristics of English and Limited English Proficient (LEP) Patients $(\mathrm{N}=4,224)$

\begin{tabular}{|c|c|c|c|}
\hline \multirow[t]{2}{*}{ Characteristics } & \multirow{2}{*}{$\begin{array}{l}\begin{array}{l}\text { English } \\
(\mathbf{N}=3660)\end{array} \\
\mathbf{N}(\%)\end{array}$} & \multirow{2}{*}{$\begin{array}{l}\begin{array}{l}\text { LEP } \\
(N=564)\end{array} \\
N(\%)\end{array}$} & \multirow[t]{2}{*}{ p value } \\
\hline & & & \\
\hline Age (Mean \pm SD) & $64.9 \pm 17.7$ & $66.6 \pm 15.2$ & 0.03 \\
\hline Gender & & & 0.04 \\
\hline Male & $1,530(41.8)$ & $210(37.2)$ & \\
\hline Female & $2,130(58.2)$ & $354(62.8)$ & \\
\hline Race /Ethnicity & & & $<0.0001$ \\
\hline White & $2,430(66.4)$ & $87(15.4)$ & \\
\hline Black & $972(26.6)$ & $51(9.0)$ & \\
\hline Hispanic & $165(4.5)$ & $381(67.6)$ & \\
\hline Asian & $39(1.1)$ & $34(6.0)$ & \\
\hline Other & $54(1.5)$ & $11(2.0)$ & \\
\hline Insurance & & & $<0.0001$ \\
\hline Commercial & $1,413(38.6)$ & $105(18.6)$ & \\
\hline Medicaid & $309(8.4)$ & $152(27.0)$ & \\
\hline Medicare & $1,747(47.7)$ & $278(49.3)$ & \\
\hline Self-Pay/Other & $191(5.2)$ & $29(5.1)$ & \\
\hline \multicolumn{4}{|l|}{ Hospitalist Service } \\
\hline Yes & $2,859(78.1)$ & $444(78.7)$ & 0.75 \\
\hline No & $801(21.9)$ & $120(21.3)$ & \\
\hline $\begin{array}{c}\text { Charlson Score } \\
(\text { Mean } \pm \text { SD) }\end{array}$ & $2.4 \pm 2.5$ & $2.5 \pm 2.3$ & 0.007 \\
\hline $\begin{array}{l}\text { Length of Stay } \\
(\text { Mean } \pm \text { SD) }\end{array}$ & $6.15 \pm 5.7$ & $5.24 \pm 4.5$ & $<0.0001$ \\
\hline $\begin{array}{l}\text { 30-Day Readmission } \\
\text { Rate }\end{array}$ & $669(18.3)$ & $96(17.0)$ & 0.47 \\
\hline $\begin{array}{l}\text { ED Utilization After } \\
\text { Discharge }\end{array}$ & $729(19.9)$ & $112(19.9)$ & 0.97 \\
\hline Discharge Diagnosis & & & 0.04 \\
\hline Cardiac & $386(10.6)$ & 77 (13.7) & \\
\hline Pneumonia & $263(7.2)$ & $37(6.6)$ & \\
\hline Urinary Infection & $136(3.7)$ & $15(2.7)$ & \\
\hline Renal & $184(5.0)$ & $32(5.7)$ & \\
\hline Respiratory & $146(4.0)$ & $24(4.3)$ & \\
\hline Pulmonary Embolism & $63(1.7)$ & $6(1.1)$ & \\
\hline Pancreatitis & $122(3.3)$ & $7(1.2)$ & \\
\hline Other & $2,360(64.5)$ & $366(64.9)$ & \\
\hline \multicolumn{4}{|l|}{ Language Category } \\
\hline No Interpreter & - & $371(65.8)$ & - \\
\hline $\begin{array}{l}\text { Interpreter w/ } \\
\text { Hospitalist Present }\end{array}$ & - & $27(4.8)$ & - \\
\hline $\begin{array}{l}\text { Interpreter w/ } \\
\text { Physician Present }\end{array}$ & - & $71(12.6)$ & - \\
\hline $\begin{array}{l}\text { Interpreter w/ } \\
\text { No Physician Present }\end{array}$ & - & $95(16.8)$ & - \\
\hline
\end{tabular}

patients $(10.4 \%)(\mathrm{p}=0.19)$. Patients who were readmitted within 30 days were more likely to be younger, White and have Medicare insurance (Table 2). Compared to those not readmitted, readmitted patients had a higher Charlson Score and a longer length of stay, but there were no significant differences by language category. Similarly, patients who utilized the ED 30 days after discharge were more likely to be younger, White, and have Medicare insurance (Table 3). In addition, these patients had a higher Charlson score and had a longer LOS, but there were no significant differences by language category.

\section{Mutltivariable Analyses of Hospital Outcomes}

Among all patients in the study, those with a higher Charlson scores had a slightly higher LOS, an increased readmission rate, and an increase in ED utilization (Table 4). Those with
Table 2 Patient Characteristics and 30-Day Readmission

\begin{tabular}{|c|c|c|c|}
\hline \multirow[t]{2}{*}{ Characteristics } & \multirow{2}{*}{$\begin{array}{l}\text { Not } \\
\text { Readmitted } \\
(3,459) \\
\text { N (\%) }\end{array}$} & \multirow{2}{*}{$\begin{array}{l}\begin{array}{l}\text { Readmitted } \\
\text { (765) }\end{array} \\
\text { N (\%) }\end{array}$} & \multirow[t]{2}{*}{ p Value } \\
\hline & & & \\
\hline Age $($ Mean \pm SD $)$ & $65.4 \pm 17.4$ & $63.8 \pm 17.6$ & 0.02 \\
\hline Gender & & & 0.68 \\
\hline Male & $1,430(41.3)$ & $310(40.5)$ & \\
\hline Female & $2,029(58.7)$ & $455(59.5)$ & \\
\hline Race & & & 0.01 \\
\hline White & $2,053(59.4)$ & $464(60.7)$ & \\
\hline Black & $843(24.4)$ & $180(23.5)$ & \\
\hline Hispanic & $440(12.7)$ & $106(13.9)$ & \\
\hline Asian & $59(1.7)$ & $14(1.8)$ & \\
\hline Other & 64 (1.9) & $1(0.1)$ & \\
\hline Insurance & & & 0.002 \\
\hline Commercial & $1,273(36.9)$ & $245(32.0)$ & \\
\hline Medicaid & $355(10.3)$ & $106(13.9)$ & \\
\hline Medicare & $1,642(47.5)$ & $383(50.1)$ & \\
\hline Self-Pay/Other & $189(5.5)$ & $31(4.1)$ & \\
\hline Hospitalist Service & & & 0.13 \\
\hline Yes & $2,689(77.7)$ & $614(80.3)$ & \\
\hline No & $770(22.3)$ & $151(19.7)$ & \\
\hline $\begin{array}{c}\text { Charlson Score } \\
(\text { Mean } \pm \text { SD) }\end{array}$ & $2.2 \pm 2.4$ & $3.0 \pm 2.6$ & $<0.001$ \\
\hline $\begin{array}{l}\text { Length of Stay } \\
(\text { Mean } \pm \text { SD) }\end{array}$ & $5.8 \pm 5.1$ & $6.9 \pm 7.1$ & $<0.001$ \\
\hline Discharge Diagnosis & & & 0.01 \\
\hline Cardiac & 385 & $78(10.2)$ & \\
\hline Pneumonia & $251(7.3)$ & $49(6.4)$ & \\
\hline Urinary Infection & $123(3.6)$ & $28(3.7)$ & \\
\hline Renal & $174(5.0)$ & $42(5.5)$ & \\
\hline Respiratory & $148(4.3)$ & $22(2.9)$ & \\
\hline Pulmonary & $59(1.7)$ & $10(1.3)$ & \\
\hline Embolism & & & \\
\hline Pancreatitis & $90(2.6)$ & $39(5.1)$ & \\
\hline Other & $2,229(64.4)$ & $497(65.0)$ & \\
\hline Language Category & & & 0.90 \\
\hline No Interpreter & $307(8.9)$ & $64(8.4)$ & \\
\hline $\begin{array}{l}\text { Interpreter w/ } \\
\text { Hospitalist Present }\end{array}$ & $22(0.6)$ & $5(0.7)$ & \\
\hline $\begin{array}{l}\text { Interpreter w/ } \\
\text { Physician Present }\end{array}$ & $60(1.7)$ & $11(1.4)$ & \\
\hline $\begin{array}{l}\text { Physician Present } \\
\text { Interpreter w/ No }\end{array}$ & $79(2.3)$ & $16(2.1)$ & \\
\hline Physician Present & & & \\
\hline English Speaker & 2,991 (86.5) & $669(87.5)$ & \\
\hline
\end{tabular}

Medicaid had an increased readmissions rate and ED utilization. Medicare patients had a longer LOS, an increased readmissions rate and ED utilization. Several diagnoses had significantly shorter LOS, while only acute pancreatitis had higher readmission rates and ED utilization. Stratified adjusted analyses by Charlson score $(0-1,2-4,5+)$ for each of the service utilization outcomes did not show differences between LEP and English speaking patients with similar Charlson scores.

Compared to English speakers in multivariable models, LEP patients with no interpreters and those who had interpreter use with a non-physician had significantly shorter LOS (Table 4). Compared to LEP patients with no interpreter use, LEP patients who had a physician use an interpreter (both nonhospitalist and hospitalist) had odds for a longer LOS, but there was no difference in odds of readmission or ED utilization (Table 5). LEP patients with a higher Charlson scores had a marginally statistically significant higher LOS and 
Table 3 Patient Characteristics and 30-Day ED Utilization After Discharge

\begin{tabular}{|c|c|c|c|}
\hline \multirow[t]{2}{*}{ Characteristics } & \multirow{2}{*}{$\begin{array}{l}\text { No ED } \\
\text { Visits(3383) } \\
\text { N (\%) }\end{array}$} & \multirow{2}{*}{$\begin{array}{l}\begin{array}{l}\text { ED Visited } \\
(841)\end{array} \\
\text { N (\%) }\end{array}$} & \multirow[t]{2}{*}{ p Value } \\
\hline & & & \\
\hline Age $($ Mean \pm SD $)$ & $65.5 \pm 17.3$ & $63.6 \pm 17.8$ & \\
\hline Gender & & & 0.29 \\
\hline Male & $1,407(41.6)$ & $333(39.6)$ & \\
\hline Female & $1,976(58.4)$ & $508(60.4)$ & \\
\hline Race & & & 0.002 \\
\hline White & $2,035(60.2)$ & $482(57.3)$ & \\
\hline Black & $806(23.9)$ & $217(25.8)$ & \\
\hline Hispanic & $420(12.4)$ & $126(15.0)$ & \\
\hline Asian & $59(1.7)$ & $14(1.7)$ & \\
\hline Other & $63(1.9)$ & $2(0.2)$ & \\
\hline Insurance & & & $<0.0001$ \\
\hline Commercial & $1,268(37.5)$ & $250(29.7)$ & \\
\hline Medicaid & $334(9.9)$ & $127(15.1)$ & \\
\hline Medicare & $1,599(47.3)$ & $426(50.7)$ & \\
\hline Self-Pay/Other & $182(5.4)$ & $38(4.5)$ & \\
\hline Hospitalist Service & & & 0.15 \\
\hline Yes & $2,630(77.7)$ & $673(80.0)$ & \\
\hline No & $753(22.3)$ & $168(20.0)$ & \\
\hline $\begin{array}{l}\text { Charlson Score } \\
(\mathrm{Mean} \pm \mathrm{SD})\end{array}$ & $2.3 \pm 2.4$ & $2.9 \pm 2.6$ & $<0.0001$ \\
\hline $\begin{array}{l}\text { Length of Stay } \\
(\text { Mean } \pm \text { SD) }\end{array}$ & $5.9 \pm 5.1$ & $6.6 \pm 7.0$ & 0.02 \\
\hline Discharge Diagnosis & & & 0.02 \\
\hline Cardiac & $378(11.2)$ & $85(10.1)$ & \\
\hline Pneumonia & $243(7.2)$ & $57(6.8)$ & \\
\hline Urinary Infection & $118(3.5)$ & $33(3.9)$ & \\
\hline Renal & $167(4.9)$ & $49(5.8)$ & \\
\hline Respiratory & $142(4.2)$ & $28(3.3)$ & \\
\hline Pulmonary Embolism & $56(1.7)$ & $13(1.6)$ & \\
\hline Pancreatitis & $87(2.6)$ & $42(5.0)$ & \\
\hline Other & $2,192(64.8)$ & $534(63.5)$ & \\
\hline Language Category & & & 0.64 \\
\hline No Interpreter & $303(9.0)$ & $68(8.1)$ & \\
\hline Interpreter w/ & $19(0.6)$ & $8(1.0)$ & \\
\hline Hospitalist Present & & & \\
\hline Interpreter w/ & $56(1.7)$ & $15(1.8)$ & \\
\hline Physician Present & & & \\
\hline Interpreter $\mathrm{w} / \mathrm{No}$ & $74(2.2)$ & $21(2.5)$ & \\
\hline $\begin{array}{l}\text { Physician Present } \\
\text { English Speaker }\end{array}$ & & & \\
\hline English Speaker & $2,931(86.6)$ & $729(86.7)$ & \\
\hline
\end{tabular}

readmission rate. Those with Medicaid and Medicare insurance had an increased readmissions rate and an increase in ED utilization, while self-pay patients had shorter LOS. LEP patients with cardiac and respiratory discharge diagnoses had significantly shorter LOS.

\section{DISCUSSION}

Our study has several important findings concerning inpatient interpreter use defined as at least one interpreted session during a hospital stay. First, our results indicate that in a well resourced academic hospital, use of interpreters by clinical staff remains highly variable, with $66 \%$ of LEP patients having no interpreter use during the inpatient clinical encounter. Secondly, compared to English speakers, LEP patients who did not have an interpreter had a shorter LOS. However, LEP patients who had a physician present with interpreter use had longer LOS compared to LEP patients without an interpreter. Finally, there were no differences in 30-day post discharge ED visits or readmission rates for LEP patients compared to English speaking patients. Similarly there were no differences in these two outcomes among LEP patients, whether or not an interpreter was used.

Similar to all prior inpatient studies we found a low use of interpreters by hospital clinicians. ${ }^{5,6,21}$ Federal law, the Institute of Medicine (IOM), and hospital guidelines, including the Joint Commission standards, all recommend the routine use of professional interpreters during clinical encounters. ${ }^{3,22-24}$ It is possible that our low rate of interpreter use is due to the use of nonprofessional interpreters (i.e., family members or friends) and/or clinical encounters with bilingual physicians or other clinical staff and/or even LEP patients relying on their own limited English language abilities. ${ }^{21}$ High quality clinical care and patient safety are compromised when there are communication barriers. The use of nonprofessional interpreters is known to cause an increase in interpreter errors that can potentially harm the patient, through the misinterpretation of information ${ }^{7,25,26}$ and alteration of key patient details. ${ }^{27}$ One study found that there was a significantly higher proportion of errors with potential clinical consequences when ad hoc interpreters or no interpreters were used compared to professional interpreters. $^{7}$

Clinicians may be 'getting by' without interpreters for a variety of reasons, including a perceived increase in clinical encounter time with the use of interpreters. ${ }^{4,21,28}$ There are several high quality studies that demonstrate no clinical encounter time increase with in-person hospital interpreters. ${ }^{14,29}$ One important time motion study in a primary care clinic found no difference in visit time with and without interpreters. ${ }^{30}$ However, almost all physicians perceived and reported an increase in time. Physicians may attempt to use their nonfluent language skills in order to save time. However, it is not a given that self-identified bilinguals are sufficiently bilingual or competent in medical terminology. The use of nonformally tested bilingual staff has been demonstrated to lead to low quality communication secondary to errors. ${ }^{4,21,28}$

LOS is a frequently used measure to assess the efficiency of hospital care delivery. ${ }^{31}$ Our study provides unique and complex findings about interpreter use and LOS. We found evidence that compared to English speakers, LEP patients with no interpreters and those who had interpreter use without a physician present had a shorter LOS. As a measure of efficiency, LEP patients without interpreters and those with an interpreter without a physician present may be receiving appropriate and timely medical care. LEP status represents a continuum of language ability, and these patients may have more English ability than is apparent or these patients may have very involved family members or friends at the bedside. This appropriate increase in efficiency (i.e., shorter LOS) is supported by the finding of no differences in 30-day ED utilization or readmission rates compared to English speakers. However, we also found that among LEP patients, when compared to those with no interpreter use, those who had a physician present with interpreter use (both hospitalist and non- 
Table 4 Multivariate Models of the Association of Hospital Outcomes with Physician Use of Interpreters $(\mathrm{N}=4,224)$

\begin{tabular}{|c|c|c|c|}
\hline \multirow[t]{2}{*}{ Characteristics } & Length of Stay & Readmission & ED Utilization \\
\hline & $\begin{array}{l}\text { Odds Ratio } \\
\text { [95\% Confidence Interval] }\end{array}$ & $\begin{array}{l}\text { Odds Ratio } \\
\text { [95\% Confidence Interval] }\end{array}$ & $\begin{array}{l}\text { Odds Ratio } \\
\text { [95\% Confidence Interval] }\end{array}$ \\
\hline English Speakers & - & - & - \\
\hline No Interpreter & $0.80[0.73-0.87]$ & $0.86[0.62-1.19]$ & $0.81[0.63-1.04]$ \\
\hline Interpreter w/ Hospitalist Present & $1.12[0.92-1.37]$ & $1.07[0.46-2.52]$ & $1.64[0.65-4.12]$ \\
\hline Interpreter w/ Physician Present & $1.14[0.89-1.45]$ & $0.78[0.40-1.52]$ & $1.01[0.56-1.81]$ \\
\hline Interpreter w/ No Physician Present & $0.77[0.67-0.89]$ & $0.91[0.53-1.58]$ & $1.08[0.67-1.73]$ \\
\hline Age & $1.00[1.00-1.00]$ & $0.99[0.99-1.00]$ & $0.99[0.99-1.00]$ \\
\hline Female & $0.99[0.93-1.05]$ & $1.08[0.92-1.26]$ & $1.10[0.93-1.30]$ \\
\hline Charlson Score & $1.05[1.03-1.06]$ & $1.10[1.07-1.13]$ & $1.08[1.05-1.12]$ \\
\hline \multicolumn{4}{|l|}{ Insurance } \\
\hline Medicaid & $1.02[0.90-1.15]$ & $1.36[1.00-1.84]$ & $1.66[1.23-2.25]$ \\
\hline Medicare & $1.05[0.99-1.12]$ & $1.24[1.04-1.47]$ & $1.41[1.20-1.65]$ \\
\hline Other & $1.01[0.89-1.15]$ & $0.85[0.56-1.28]$ & $1.12[0.72-1.74]$ \\
\hline Self-Pay & $1.01[0.58-1.76]$ & $0.86[0.28-2.63]$ & $0.55[0.13-2.27]$ \\
\hline Commercial & - & - & - \\
\hline Hospitalist Service & $1.01[0.95-1.08]$ & $1.15[0.93-1.41]$ & $1.16[0.97-1.39]$ \\
\hline \multicolumn{4}{|l|}{ Discharge Diagnosis } \\
\hline Cardiac & $0.63[0.60-0.67]$ & $0.95[0.73-1.22]$ & $0.95[0.76-1.19]$ \\
\hline Pneumonia & $0.96[0.87-1.05]$ & $0.83[0.59-1.19]$ & $0.91[0.66-1.25]$ \\
\hline Urinary Infection & $0.79[0.71-0.87]$ & $1.01[0.66-1.52]$ & $1.14[0.78-1.65]$ \\
\hline Renal & $0.80[0.70-0.91]$ & $1.04[0.79-1.37]$ & $1.17[0.87-1.58]$ \\
\hline Respiratory & $0.72[0.65-0.79]$ & $0.61[0.36-1.04]$ & $0.72[0.45-1.16]$ \\
\hline Pulmonary Embolism & $0.84[0.74-0.96]$ & $0.87[0.48-1.58]$ & $1.08[0.59-1.95]$ \\
\hline Acute Pancreatitis & $0.99[0.83-1.18]$ & $1.88[1.36-2.59]$ & $1.89[1.32-2.71]$ \\
\hline Other & - & - & - \\
\hline
\end{tabular}

hospitalist) had a longer LOS in fully adjusted models. This may reflect physician selective use of interpreters for more medically complicated patients. Indeed, our analyses found that patients with an interpreter and an MD present had the highest Charlson score, while those with an interpreter and a non MD present had the lowest comorbidity score.

There are several possible reasons for the observed increased LOS with physician use of interpreters. Several studies show that when LEP patients have access to an interpreter, they actually have increased utilization of services and have improved disease-specific process measures/testing and outcomes. $^{5}$ It is possible that the sicker LEP patients required more appropriate medical care or that sicker LEP patients lacked a regular source of medical care, therefore getting most of their workup as an inpatient. In addition, one study has shown that the LOS for LEP patients was longer for seven of 23 medical and surgical conditions. ${ }^{15}$ Finally, there is evidence that high quality care takes more time. ${ }^{32,33}$ A greater amount of clinical items can be addressed over a longer period of time, which then leads to a higher quality of care. Thus, an increase

Table 5 Multivariate Models of the Association of Hospital Outcomes with Physician Use of Interpreters Among LEP Only (N=564)

\begin{tabular}{|c|c|c|c|}
\hline \multirow[t]{2}{*}{ Characteristics } & \multirow{2}{*}{$\begin{array}{l}\text { Length of Stay } \\
\text { Odds Ratio } \\
\text { [95\% Confidence Interval] }\end{array}$} & \multirow{2}{*}{$\begin{array}{l}\text { Readmission } \\
\text { Odds Ratio } \\
\text { [95\% Confidence Interval] }\end{array}$} & \multirow{2}{*}{$\begin{array}{l}\text { ED Utilization } \\
\text { Odds Ratio } \\
\text { [95\% Confidence Interval] }\end{array}$} \\
\hline & & & \\
\hline No Interpreter & - & - & - \\
\hline Interpreter w/ Hospitalist Present & $1.30[1.08-1.57]$ & $1.04[0.44-2.45]$ & $2.04[0.88-4.74]$ \\
\hline Interpreter w/ Physician Present & $1.41[1.08-1.84]$ & $1.01[0.53-1.94]$ & $1.19[0.62-2.28]$ \\
\hline Interpreter w/ No Physician Present & $0.94[0.81-1.09]$ & $1.20[0.65-2.21]$ & $1.25[0.70-2.21]$ \\
\hline Age & $1.00[0.99-1.00]$ & $0.99[0.97-1.01]$ & $0.99[0.97-1.01]$ \\
\hline Female & $1.01[0.89-1.14]$ & $0.96[0.51-1.77]$ & $1.01[0.58-1.74]$ \\
\hline Charlson Score & $1.02[1.00-1.05]$ & $1.07[0.98-1.17]$ & $0.98[0.89-1.07]$ \\
\hline \multicolumn{4}{|l|}{ Insurance } \\
\hline Medicaid & $0.94[0.80-1.12]$ & $2.45[1.07-5.60]$ & $2.78[1.24-6.26]$ \\
\hline Medicare & $1.11[0.95-1.31]$ & $2.72[1.20-6.18]$ & $2.69[1.25-5.79]$ \\
\hline Self-Pay/Other & $0.76[0.60-0.95]$ & $0.84[0.17-4.15]$ & $1.64[0.46-5.82]$ \\
\hline Commercial & - & - & - \\
\hline Hospitalist Service & $1.00[0.85-1.18]$ & $1.31[0.68-2.52]$ & $0.85[0.48-1.49]$ \\
\hline \multicolumn{4}{|l|}{ Discharge Diagnosis } \\
\hline Cardiac & $0.63[0.53-0.75]$ & $0.87[0.46-1.64]$ & $1.08[0.63-1.87]$ \\
\hline Pneumonia & $0.99[0.75-1.31]$ & $0.88[0.40-1.96]$ & $0.65[0.29-1.43]$ \\
\hline Urinary Infection & $1.07[0.69-1.66]$ & $0.82[0.22-3.07]$ & $0.60[0.15-2.45]$ \\
\hline Renal & $1.03[0.73-1.44]$ & $1.01[0.33-3.05]$ & $1.65[0.61-4.43]$ \\
\hline Respiratory & $0.66[0.52-0.85]$ & $0.53[0.15-1.93]$ & $0.47[0.14-1.54]$ \\
\hline Pulmonary Embolism & $0.84[0.45-1.60]$ & $1.29[0.11-14.83]$ & $2.35[0.28-19.47]$ \\
\hline Acute Pancreatitis & $1.11[0.61-2.04]$ & $3.61[0.59-22.00]$ & $1.74[0.29-10.55]$ \\
\hline Other & - & - & - \\
\hline
\end{tabular}


in LOS (i.e., a decrease in measured efficiency) may be a marker of high quality care. Our analyses demonstrated that there were no differences in outcomes between English speakers and LEP patients in adjusted stratified analyses by Charlson score, suggesting that regardless of language ability, equally ill patients have similar outcomes.

Finally, compared to English speakers, our study found no differences in ED utilization and readmission within 30 days for both any and same diagnoses. In addition, among LEP patients, increased Charlson score, Medicaid and Medicare insurance status is associated with readmission within 30 days and with an increased utilization within 30 days. Our study currently has only one other published paper for comparison. Jacobs et al. found no difference in LOS and 3-month ED utilization or readmission rates with data from a large urban public hospital. ${ }^{14}$ However, it is difficult to contextualize these results with our findings, because the study is a three-arm study comparing English speakers to two groups of Spanish speakers with access to an enhanced interpreter or the usual interpreter services. Their findings may not be immediately applicable in other settings. In addition, unlike our study, Jacobs et al. was able to account for the effect of formally tested Spanish clinicians in situations where a professional interpreter was not used.

Our study has several important policy implications for future research on the use of interpreters in the inpatient setting. First, our findings highlight the patient heterogeneity that underlies LEP status. The LEP status continuum may be affected by other important factors, such as the patient's length of residence in the US and patients' preferences for interpreter use. Future studies will need to account for this complexity, allowing for more precise and accurate measure of LEP status and interpreter need. Second, inpatient care is a continuum of clinical care encounters with both clinical and non-clinical staff. All patient interactions with clinical providers should document whether an interpreter or a bilingual clinician was used in order to allow quality assessment both in real time and retrospectively. It is likely not enough to have interpreter use only at the time of admission or possibly only at discharge. Finally, increasing the availability and use of professional interpreter throughout the hospital stay is essential. This will also require educating physicians about the quality of available interpretation modalities. Recent studies have demonstrated that remote telephone or video conferencing modes are as effective as in-person interpretation as measured by communication quality and patient satisfaction. ${ }^{34-37}$ Use of these non in-person methods should be encouraged in the hospital setting, where in-person interpretation may not be readily available throughout the 24-hour hospital continuum of care.

There are several limitations to our study. First, our study population was obtained from one urban large tertiary academic teaching hospital and may not be generalizable to other settings. Second, our outcome measures may not have fully captured all 30-day ED utilization or readmissions secondary to off-site utilization, and this data is usually not captured in most urban hospital markets in the US. Although we accounted for many significant confounders, we were unable to include other important patient characteristics that could have impacted our outcomes, such as educational attainment, social support and having a usual place of care. ${ }^{38,39}$ Our data does not allow clear identification of when during the inpatient stay an interpreter was used, or if multiple interpreter encounters occurred. Finally, our study is limited by measurement of one interpreted session per inpatient stay, and this may impact our outcome measure results, as this minimal definition may not be strong enough to impact our outcome measures.

\section{CONCLUSION}

This study was able to assess the effect of the use of at least one interpreter encounter on LEP patients' inpatient outcomes, which has only been studied in two prior studies. Future studies will need to disaggregate the continuum of inpatient care experiences for LEP patients. Studies are needed to focus on the impact of interpreters at particularly sensitive communication moments, such as discharge, to assess the effect on patient outcomes. Being able to assess the dynamic of clinician-patient-interpreter communication is essential for understanding inpatient processes of care for LEP patients and their outcomes, thus paving the way for eliminating disparities.

Acknowledgements: Dr. López thanks the RWJ Foundation Harold Amos Faculty Development Program and NIDDK 1K23DK098280-01. Dr. Hicks effort was supported by an Institutional Development Award (IDeA) from the National Institute of General Medical Sciences of the National Institutes of Health under grant number U54GM104941 (PI: Binder-Macleod). These funding bodies were not involved in the design, data collection, analysis, interpretation or the preparation of the manuscript.

Conflict of Interest: The authors declare that they do not have a conflict of interest.

Corresponding Author: Lenny López, MD, MPH; Department of General Medicine, Mongan Institute for Health Policy, and Disparities Solutions CenterMassachusetts General Hospital, Boston, MA, USA (e-mail: llopez1@partners.org).

\section{REFERENCES}

1. United States Census Bureau. Language spoken at home by ability to speak English for the population 5 years and over. Available at: http:// factfinder2.census.gov/faces/tableservices/jsf/pages/productview.xhtml? pid=ACS_11_1YR_B16001\&prodType=table. Accessed January 13, 2015.

2. Bernstein R. U.S. Census Bureau projections show a slower growing, older, more diverse nation a half century from now. United States Census Bureau Newsroom [Internet]. 2012 December 12. Available at: https:// www.census.gov/newsroom/releases/archives/population/cb12-243. html. Accessed January 13, 2015

3. Wilson-Stronks A, Galvez E. Hospitals, language and culture: A snapshot of the nation. Exploring cultural and linguistic services in the nation's hospitals: A report of findings. The Joint Commission and The California Endowment 2007. 
4. Diamond LC, Schenker Y, Curry L, Bradley EH, Fernandez A. Getting by: underuse of interpreters by resident physicians. J Gen Intern Med. 2009;24(2):256-62. doi:10.1007/s11606-008-0875-7.

5. Karliner LS, Jacobs EA, Chen AH, Mutha S. Do professional interpreters improve clinical care for patients with limited English proficiency? A systematic review of the literature. Health Serv Res. 2007;42(2):727-54. doi:10.1111/j.1475-6773.2006.00629.x.

6. Flores G. The impact of medical interpreter services on the quality of health care: a systematic review. Med Care Res Rev. 2005;62(3):255-99. doi: $10.1177 / 1077558705275416$.

7. Flores G, Abreu M, Barone CP, Bachur R, Lin H. Errors of medical interpretation and their potential clinical consequences: a comparison of professional versus ad hoc versus no interpreters. Ann Emerg Med. 2012;60(5):545-53. doi:10.1016/j.annemergmed.2012.01.025.

8. Cohen AL, Rivara F, Marcuse EK, McPhillips H, Davis R. Are language barriers associated with serious medical events in hospitalized pediatric patients? Pediatrics. 2005;116(3):575-9. doi:10.1542/peds. 2005-0521.

9. Divi C, Koss RG, Schmaltz SP, Loeb JM. Language proficiency and adverse events in US hospitals: a pilot study. Int $\mathrm{J}$ Qual Health Care. 2007;19(2):60-7. doi:10.1093/intqhe/mzl069.

10. Institute of Medicine. Crossing the Quality Chasm: A New Health System for the 21st Century 2001.

11. Ramirez D, Engel KG, Tang TS. Language interpreter utilization in the emergency department setting: a clinical review. J Health Care Poor Underserved. 2008;19(2):352-62. doi:10.1353/hpu.0.0019.

12. Lindholm M, Hargraves JL, Ferguson WJ, Reed G. Professional language interpretation and inpatient length of stay and readmission rates. J Gen Intern Med. 2012;27(10):1294-9. doi:10.1007/s11606-012-2041-5.

13. Grubbs V, Bibbins-Domingo $\mathbf{K}$, Fernandez A, Chattopadhyay A, Bindman AB. Acute myocardial infarction length of stay and hospital mortality are not associated with language preference. J Gen Intern Med. 2008;23(2):190-4. doi:10.1007/s11606-007-0459-y.

14. Jacobs EA, Sadowski LS, Rathouz PJ. The impact of an enhanced interpreter service intervention on hospital costs and patient satisfaction. J Gen Intern Med. 2007;22(Suppl 2):306-11. doi:10.1007/s11606-007-0357-3.

15. John-Baptiste A, Naglie G, Tomlinson G, et al. The effect of English language proficiency on length of stay and in-hospital mortality. J Gen Intern Med. 2004;19(3):221-8. doi:10.1111/j.1525-1497.2004.21205.x.

16. Karliner LS, Kim SE, Meltzer DO, Auerbach AD. Influence of language barriers on outcomes of hospital care for general medicine inpatients. J Hosp Med. 2010;5(5):276-82. doi:10.1002/jhm.658.

17. Smedley BD, Stith AY, Nelson AR. editors (Institute of Medicine). Unequal treatment: confronting racial and ethnic disparities in health care (with CD). Washington, DC: National Academies Press; 2003.

18. Nalichowski R, Keogh D, Chueh HC, Murphy SN. Calculating the benefits of a Research Patient Data Repository. AMIA Annual Symposium proceedings/AMIA Symposium. AMIA Symposium 2006:1044.

19. The Official Website of the Executive Office of Health and Human Services. Interpreter services. Available at: http://www.mass.gov/eohhs/gov/departments/dph/programs/admin/health-equity/interpreter-services. html. Accessed January 13, 2015.

20. Official Website of the City of Boston. Demographic report. Available at: http://www.cityofboston.gov/newbostonians/publications.asp. Accessed January 13, 2015.

21. Schenker Y, Perez-Stable EJ, Nickleach D, Karliner LS. Patterns of interpreter use for hospitalized patients with limited English proficiency. J Gen Intern Med. 2011:26(7):712-7. doi:10.1007/s11606-010-1619-z.
22. U.S. Department of Health and Human Services, Office of Minority Health. National standards for culturally and linguistically appropriate services in health care. Washington, DC; 2001.

23. Ulmer C, McFadden B, Nerenz DR. editors (Institute of Medicine). Race, ethnicity, and language data: standardization for health care quality improvement. Washington, DC: National Academies Press; 2009.

24. Equal Employment Opportunity Program (US). Title VI, Prohibition against national origin discrimination affecting limited english proficient persons. Final guidance. Fed Regist. 2004;69(7):1763-8.

25. Ebden P, Carey OJ, Bhatt A, Harrison B. The bilingual consultation. Lancet. 1988;1(8581):347.

26. David RA, Rhee M. The impact of language as a barrier to effective health care in an underserved urban Hispanic community. Mt Sinai J Med. 1998;65(5-6):393-7.

27. Launer J. Taking medical histories through interpreters: practice in a Nigerian outpatient department. Br Med J. 1978;2(6142):934-5.

28. Diamond LC, Tuot DS, Karliner LS. The use of Spanish language skills by physicians and nurses: Policy implications for teaching and testing. J Gen Intern Med. 2012;27(1):117-23. doi:10.1007/s11606011-1779-5.

29. Fagan MJ, Diaz JA, Reinert SE, Sciamanna CN, Fagan DM. Impact of interpretation method on clinic visit length. J Gen Intern Med. 2003;18(8):634-8. doi:10.1046/j.1525-1497.2003.20701.x.

30. Tocher TM, Larson EB. Do physicians spend more time with non-Englishspeaking patients? J Gen Intern Med. 1999;14(5):303-9.

31. Shi L. Patient and hospital characteristics associated with average length of stay. Health Care Manag Rev. 1996;21(2):46-61.

32. Chen LM, Farwell WR, Jha AK. Primary care visit duration and quality: does good care take longer? Arch Intern Med. 2009;169(20):1866-72. doi:10.1001/archinternmed.2009.341.

33. Abbo ED, Zhang Q, Zelder M, Huang ES. The increasing number of clinical items addressed during the time of adult primary care visits. J Gen Intern Med. 2008;23(12):2058-65. doi:10.1007/s11606-0080805-8.

34. Gany F, Leng $\mathbf{J}$, Shapiro E, et al. Patient satisfaction with different interpreting methods: a randomized controlled trial. J Gen Intern Med. 2007;22(Suppl 2):312-8. doi:10.1007/s11606-007-0360-8.

35. Jacobs EA, Leos GS, Rathouz PJ, Fu P Jr. Shared networks of interpreter services, at relatively low cost, can help providers serve patients with limited english skills. Health Aff. 2011;30(10):1930-8. doi:10.1377/hlthaff. 2011.0667.

36. Karliner LS, Mutha S. Achieving quality in health care through language access services: lessons from a California public hospital. Am J Med Qual. 2010;25(1):51-9. doi:10.1177/1062860609351237.

37. Napoles AM, Santoyo-Olsson J, Karliner LS, O'Brien H, Gregorich SE, Perez-Stable EJ. Clinician ratings of interpreter mediated visits in underserved primary care settings with ad hoc, in-person professional, and video conferencing modes. J Health Care Poor Underserved. 2010;21(1):301-17. doi:10.1353/hpu.0.0269.

38. Howie-Esquivel J, Dracup K. Effect of gender, ethnicity, pulmonary disease, and symptom stability on rehospitalization in patients with heart failure. Am J Cardiol. 2007;100(7):1139-44. doi:10.1016/j.amjcard.2007. 04.061.

39. Campbell SE, Seymour DG, Primrose WR, Project A. A systematic literature review of factors affecting outcome in older medical patients admitted to hospital. Age Ageing. 2004;33(2):110-5. doi:10.1093/ageing/ afh036. 